
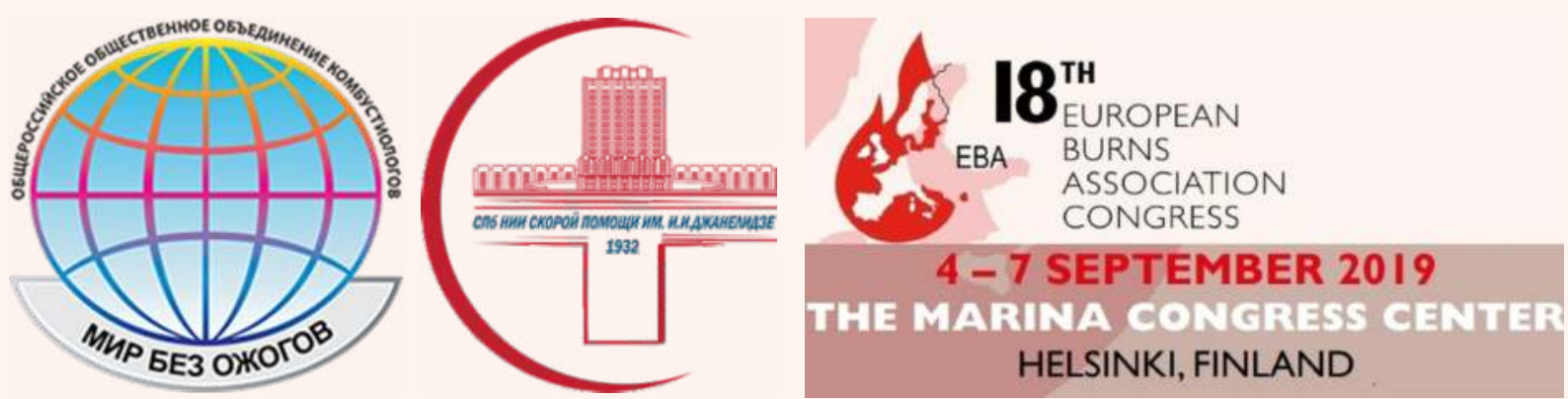

\title{
TREATMENT OF «SOFAINJURY»
}

VagnerD.O.1,2, ZinovievE.V.1, Soloshenko V.V.1, Chuvashev N.S.2

1St. Petersburg Institute of Emergency Care named after I.I. Dzhanelidze, St. Petersburg, Russia

2North-Western state medical university named after I.I. Mechnikov, Saint-Petersburg, Russia.

Objective. Smoking in bed or sofa coupled with alcohol intoxication is one of the causes of severe burns when a cigarette is dropped by the person while asleep. Due to alcohol intoxication the pain threshold decreases and consciousness is depressed. This is accompanied by the patient's long exposure to the fire with the formation of extensive and deep burns. In this case burn wounds are located in the body areas adjacent to the burned sofa. The term "sofa injury" is widely used to describe this multifactorial thermo-toxic injury.

Methods. A retrospective study included patients with burns more than $10 \%$ TBSA. The main group consisted of 60 patients who got burned as a result of accidentally burning a couchwith a cigarette. The control group included 330 patients with burns of the same areas whichwere not associated with "sofa injuries".

Results. Sofa injuries are more common in male than in female $(p=0.002)$. Alcohol intoxication significantly increases the risk of such injuries $(p=0.000)$. The age $(p=0.570)$ and TBSA $(p=0.396)$ were the same for the affected main and control groups. At the same time, FTSA in victims with "sofa injury" was larger than in control patients $(p=0.000)$. Surgical treatment of patients with "sofa trauma" begins with escharotomy, which is performed immediately after admission. The method of choice for the treatment of these patients is early excision with one-stage skin graft. But simultaneous skin grafting after an early excision is possible in no more than $42 \%$ of cases. If during the first necrectomy, radicalization of the excision cannot be achieved, a vacuum or hydrocolloid dressing should be considered as a method of temporary wound closure. Victims with a "sofa injury" need longer $(p=0.000)$ and expensive $(p=0.05)$ treatment than other categories of burned ones. However, even in spite of modern high-tech methods of treatment, mortality in this group reaches $53 \%$, which is significantly more than with any other type of thermal injury $(p=0.002)$.

Conclusion. Treatment of patients with a "sofa injury" is the longest and costly, which requires the speedy transfer of these patients to specialized burn centers. The mortality in these patients is very high which requires the introduction of modern methods of surgical treatment, the most promising of which are early excision with one-stage skin graft or NPWT with delayed skin graft on granulating wound.

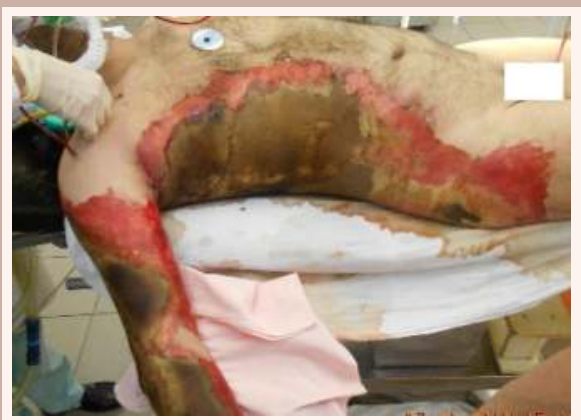

Fig. 2 Sofa injury, lesion to the trunk and upper limb

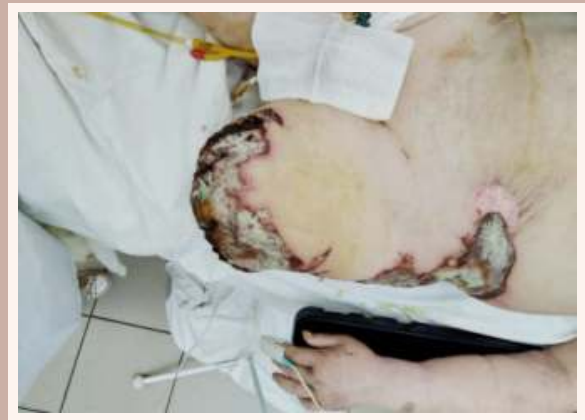

Fig. 4.2 Amputation of the left thigh

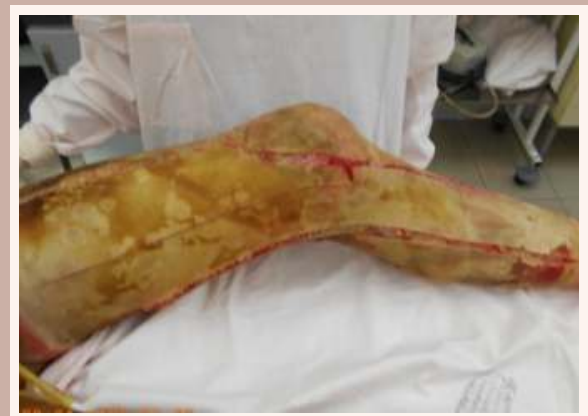

Fig. 3.1 Sofa injury, lesion to the lower limb within the skin

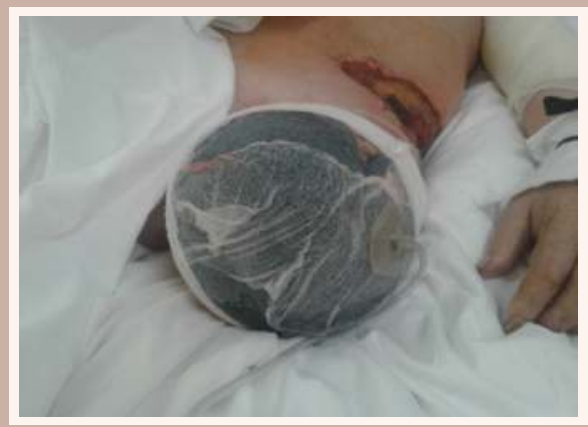

Fig. 4.3 Temporary closure of wound with NPWT-dressing

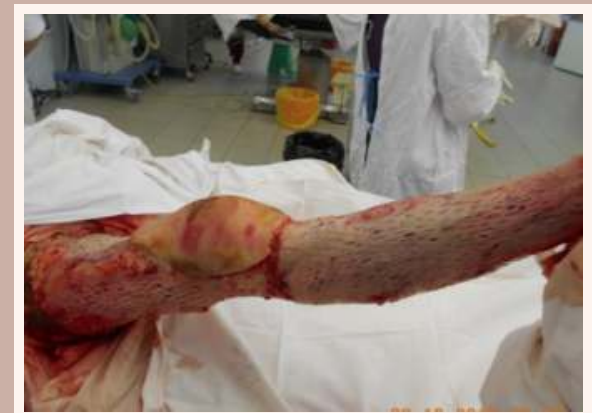

Fig. 3.2 Early excision with one-stage skin graft

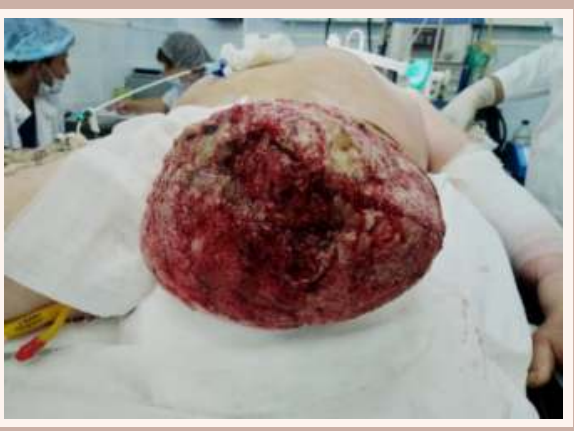

Fig. 4.4 Formation of granulations after vacuum therapy

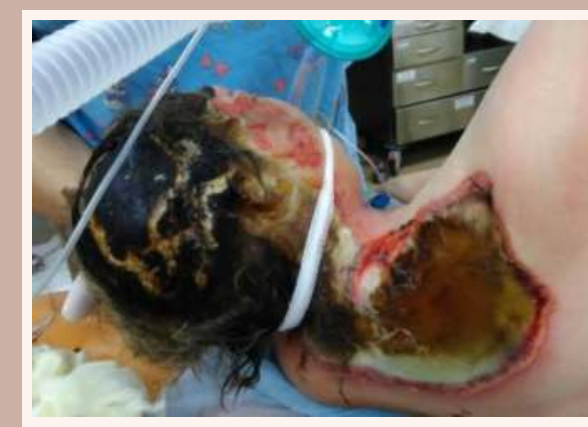

Fig. 1 Sofa injury, lesion to the head and trumk

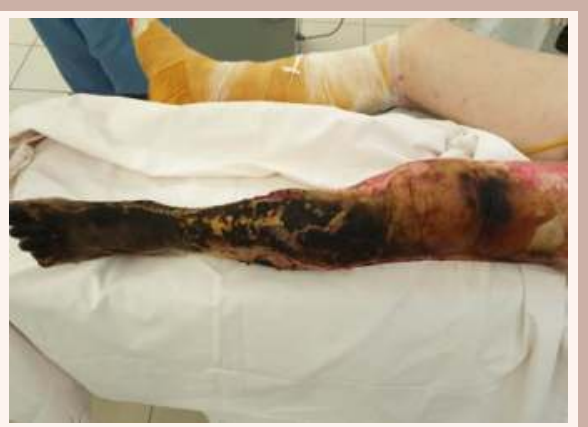

Fig. 4.1 Sofa injury, subfascial lesion of lower limb

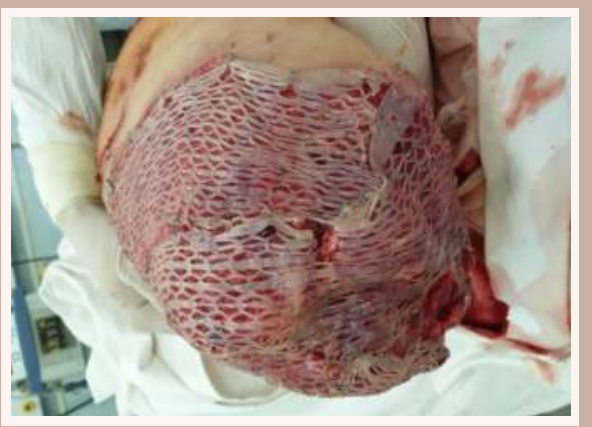

Fig. 4.5 Delayed skin graft on granulating wound 